118) for women, with $68 \%$ of men and $44 \%$ of women below the estimated average requirement (EAR). The mean WOMAC pain score at $0,12,24,36$, and 48 months of follow up was 3.5 (SD 3.8), median score 2 (IQR 0;6). After adjustment for age, gender, BMI, caloric intake, physical activity, smoking status, alcohol use, renal insufficiency, and the use of analgesics, subjects in the lowest gender-specific quintile of magnesium intake (Q1) had, on average, 1 point higher WOMAC knee pain scores than subjects in the highest quintile (Q5): Q1 $\beta$ $(95 \%$ Cl) 1.00 (0.61-1.39), Q2 0.69 (0.32-1.06), Q3 $0.25(-0.12-0.62)$, Q4 0.25 $(-0.14-0.64) ; p<0.0001$.

Conclusions: In a cohort of adults with prevalent radiographic knee osteoarthritis, magnesium intake overall was below recommended. Low magnesium intake at baseline was associated with increased pain in the affected knee over 48 months of follow up. These findings may be of growing importance as the average dietary magnesium intake for humans is declining.[3]

References:

[1] Thakur M, Dickenson AH, Baron R. Osteoarthritis pain: nociceptive or neuropathic? Nat Rev Rheumatol. 2014;10(6):374-380. doi: 10.1038/nrrheum.2014.47.

[2] Fawaz-Estrup F. The osteoarthritis initiative: an overview. Med Health R I. 2004;87(6):169-171.

[3] Rosanoff A, Weaver CM, Rude RK. Suboptimal magnesium status in the United States: are the health consequences underestimated? Nutr Rev. 2012;70(3):153-164. doi:10.1111/j.1753-4887.2011.00465.x.

Disclosure of Interest: None declared

DOI: 10.1136/annrheumdis-2017-eular.3024

\section{SAT0485 INCREASED EXPRESSION OF OSTEOPROTEGERIN IN CD271+ MULTIPOTENTIAL STROMAL CELLS FROM FEMORAL HEADS OF PATIENTS WITH HIP OSTEOARTHRITIS}

${\text { D.C. } \|^{1}{ }^{1}}^{1}$, S.M. Churchman ${ }^{1}$, J. Aderinto ${ }^{2}$, P.V. Giannoudis ${ }^{1}$, D. McGonnagle ${ }^{1}$, E. Jones ${ }^{1}$. ' Leeds Institute of Rheumatic and Musculoskeletal Medicine, University of Leeds; ${ }^{2}$ Department of Orthopaedics, Leeds Teaching Hospitals NHS Trust, Leeds, United Kingdom

Background: Osteoarthritis is a disease of the whole joint, but subchodral bone marrow lesions (BMLs) closely correlate with disease progression [1]. We have previously shown that in OA femoral heads, subchondral bone multipotential stromal cells (MSCs) were 5-fold more abundant in MRI determined BML areas where they also showed reduced mineralisation capacity and altered RANKL expression compared to non-BML areas [2]. This offered novel insight into MSC involvement in the bone remodeling process in OA.

Objectives: The current study investigated a multi-lineage gene expression profile of native CD271+ MSCs from OA femoral heads focusing on osteogenesis and bone remodeling related genes compared to CD271+ MSCs from healthy trabecular bone.

Methods: Femoral heads were obtained from 17 OA patients undergoing total hip arthroplasty. Control samples included healthy iliac crest bone from 12 patients undergoing autograft harvesting for bone reconstruction and healthy femoral neck bone from 2 patients, extracted following core decompression surgery for avascular necrosis of the femoral head. CD271+ MSCs were extracted by enzymatic digestion and purified by FACS, as previously described [3, 4]. MSC cultures were generated by standard methods [4]. Quantitative real-time PCR was performed using TaqMan assays for 12 transcripts and gene expression was normalised to HPRT1.

Results: Sorted CD271+ cells from both groups displayed gene expression profiles indicative of their steady-state osteogenic, adipogenic, bone remodeling and stromal-support functions (Table 1). Three out of 11 transcripts showed significant increases in OA: alkaline phosphatase, melanoma cell adhesion molecule and osteoprotegerin (OPG) $(p<0.05$, Mann Whitney test). The proportion of CD271 + cells in relation to total live cells was more variable but on average 2-fold higher in OA (medians of 2.3 and $5.2 \%$, respectively, not significant). Consistent with previous findings [4, 5], gene expression levels for the majority of transcripts were altered following MSC culture expansion (3 up-regulated and 8 down-regulated).

\begin{tabular}{|c|c|c|c|}
\hline \multirow{2}{*}{ Transeript } & \multicolumn{2}{|c|}{ Median (Range) } & \multirow{2}{*}{$\begin{array}{l}\text { Fold inerease in } \\
\text { OA }\left({ }^{*}=p<0.05\right)\end{array}$} \\
\hline & OA & Healthy & \\
\hline Osterix & $0.5(0.1-16.3)$ & $0.2(0.1-1.6)$ & 2.4 \\
\hline Alkatine phosphatase & $17(2.4-71.3)$ & $7.1(0.5-16.3)$ & $2,4^{*}$ \\
\hline Collagen tal & $3.1(0.2-18.7)$ & $1.1(0.4-8.9)$ & 29 \\
\hline Osteonectin & $89.8(6.1-163.1)$ & $15.3(11.3 .81 .6)$ & 5.9 \\
\hline Osteopontin & $49.2(9.6-693)$ & $46(6.9 .212 .5)$ & 1.3 \\
\hline Cadherin-11 & $15.6(1.3-37.9)$ & $5.0(3.2 .29 .2)$ & 3.1 \\
\hline Fatty acid-binding protein-4 & $1.9(0.3-10.1)$ & $0.8(0.4-11)$ & 2.5 \\
\hline $\begin{array}{l}\text { Receptor activator of buclear } \\
\text { fictor kappa-B ligand (RANKL) }\end{array}$ & $0.3(0.2-2.3)$ & $0.2(0.1-0.5)$ & 1.1 \\
\hline Osteoprotegerin (OPG) & $0.5(0.1-0.8)$ & $0.1(0.1-0.4)$ & $3.8^{\circ}$ \\
\hline Stromal cell-derived factor I & $164.6(22-1377)$ & $128.3(91.1-1956.8)$ & 1.3 \\
\hline $\begin{array}{l}\text { Melanoma cell adhesion } \\
\text { molecule }\end{array}$ & $4.5(1.0-16.3)$ & $0.9(0.9 .3 .1)$ & $5.1^{*}$ \\
\hline
\end{tabular}

Conclusions: This is the first study to show gene expression alterations in uncultured CD271+ MSCs from OA patients. A notable increase in OPG is suggestive of MSCs' bias towards inhibition of bone resorption in late hip OA and further incriminates the RANKL/OPG pathway, specifically in MSCs, in OA pathophysiology. Further studies are needed to define the role of native MSCs in lesion associated OA disease progression and to evaluate these as a possible target for treatment.

References:

[1] Goldring et al, Nat Rev Rheumatol. 2016;12:632.

[2] Campbell et al, Arthritis Rheumatol. 2016;68:1648-

[3] Jones et al., Arthritis Rheum. 2010 Jul;62:1944.

[4] Churchman et al., Arthritis Rheum. 2012 Aug;64:2632.

[5] Churchman et al., Stem Cells Int, 2017 (in press).

Disclosure of Interest: None declared

DOI: 10.1136/annrheumdis-2017-eular.5937

\section{SAT0486 CATASTROPHIZING IN OSTEOARTHRITIS OF THE KNEE: DOES THE LEQUESNE SCORE TAKE IT IN COUNT? A PROSPECTIVE STUDY}

J. Lecorney, M. Chouk, X. Guillot, C. Prati, D. Wendling, F. Verhoeven. Rhumatologie, CHRU de Besançon, Besançon, France

Background: knee osteoarthritis is a frequent matter of consultation in both general practice and rheumatology. Pain is the main symptom leading to a consultation. This painful experience can be influenced by various factors such as the anxio-depressive state or catastrophizing. Catastrophizing is known to be associated with lower analgesia results in total knee arthroplasty (1).

Objectives: The aim of this study is to evaluate the correlation between the functional status (assessed by the Lequesne score) and the catastrophizing (assessed by the Sullivan score) in patient with osteoarthritis of the knee.

Methods: We included patients consulting for knee osteoarthritis, meeting the OARSI criteria (2), one of the three general practitioners participating in the study or a rheumatologist at University Hospital. We excluded those with surgical indication. For each patient we registered the epidemiological data, the radiographic stage as well as the scores of Lequesne and Sullivan questionnaires. The correlation between the different scores were assessed with a Spearman test.

Results: A total of 100 patients were included, 50 consulting a general practitioner and 50 consulting a rheumatologist, between November 2015 and April 2016. Among these 100 patients, there were mainly women (57\%) with a mean age of $64.8 \pm 1.17$ years old. The mean radiographic stage was $2.66 \pm 0.11$ on the Kellgren and Lawrence scale. The Mean Sullivan score was $12.98 \pm 1.18$ and the mean Lequesne score was $10.46 \pm 0.47$. There was a close correlation $(r=0.3, p=0.006)$ between the Lequesne score and the radiographic scale. There was no correlation between the Sullivan score and the radiographic scale. Howerver, the correlation between the Sullivan and the Lequesne scores was modest $(r=0.47 ; p<0.0001)$ (Figure 1). Thus, the Lequesne score is correlated with the radiographic scale and the Sullivan score. When we compare the two populations, it appeared that the people consulting a rheumatologist are majority of women (72\%) and younger (62.58 \pm 1.8 VS $67,18 \pm 1.38$ years, $p<0.05$ ) and with a shorter duration of symptoms $(6.02 \pm 1.12$ VS $12.96 \pm 1.5$ Years, $p<0.001)$. In terms of radiographic and functional impairment, the two populations were similar (Kellgren score of $2.59 \pm 0.15$ VS $2.7 \pm 0.158$ and Lequesne score of $11.13 \pm 0.67$ VS $9.78 \pm 0,66$ $\mathrm{p}>0.05)$ but there was a clear difference for the Sullivan score $(16.96 \pm 1.67 \mathrm{VS}$ $9 \pm 1.4 ; p<0.001)$.

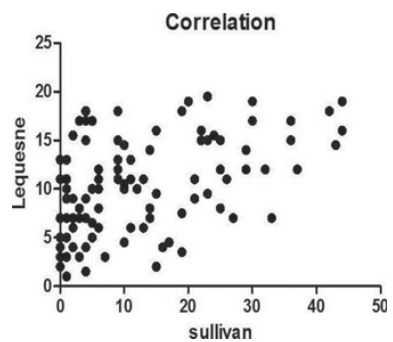

Conclusions: Our study is the first to highlight a correlation between the Sullivan and the Lequesne scores in patient consulting for osteoarthritis of the knee. Moreover, unlike the Sullivan, score the Lequesne score is correlated with the radiographic scale. The strength of this study is the inclusion of both patients consulting the general practitioner and those consulting the rheumatologist to avoid a recruitment bias. Indeed, patients consulting the rheumatologist had a catastrophizing score more important for the same functional and radiological impairment than those consulting the general practitioner. Thus, our population is representative of the global population suffering from osteoarthritis of the knee in terms of age, sex-ratio, BMI.The Lequesne score is a global score correlated with structural damage and with psychological factors like catastrophizing.

Disclosure of Interest: None declared

DOI: 10.1136/annrheumdis-2017-eular.2617 